\title{
Improved Lignin Polyurethane Properties with Lewis Acid Treatment
} Hoyong Chung* ${ }^{\dagger, \ddagger}$ and Newell R. Washburn ${ }^{\dagger, \S}$

${ }^{\dagger}$ Department of Chemistry, ${ }^{\S}$ Department of Biomedical Engineering, Carnegie Mellon University, 4400 Fifth Avenue, Pittsburgh, Pennsylvania 15213, United States

Supporting Information

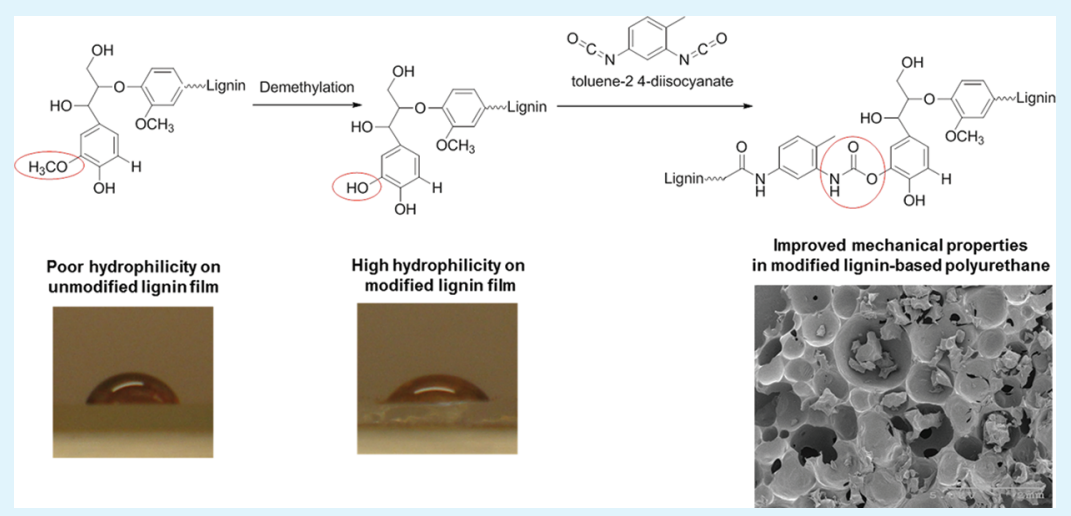

ABSTRACT: Chemical modification strategies to improve the mechanical properties of lignin-based polyurethanes are presented. We hypothesized that treatment of lignin with Lewis acids would increase the concentration of hydroxyl groups available to react with diisocyanate monomers. Under the conditions used, hydrogen bromide-catalyzed modification resulted in a $28 \%$ increase in hydroxyl group content. Associated increases in hydrophilicity of solvent-cast thin films were also recorded as evidenced by decreases in water contact angle. Polyurethanes were then prepared by first preparing a prepolymer based on mixtures of toluene-2,4-diisocyanate (TDI) and unmodified or modified lignin, then polymerization was completed through addition of polyethylene glycol (PEG), resulting in mass ratios of TDI:lignin:PEG of 43:17:40 in the compositions investigated here. The mixture of TDI and unmodified lignin resulted in a lignin powder at the bottom of the liquid, suggesting it did not react directly with TDI. However, a homogeneous solution resulted when TDI and the hydrogen bromide-treated lignin were mixed, suggesting demethylation indeed increased reactivity and resulted in better integration of lignin into the urethane network. Significant improvements in mechanical properties of modified lignin polyurethanes were observed, with a 6.5 -fold increase in modulus, which were attributed to better integration of the modified lignin into the covalent polymer network due to the higher concentration of hydroxyl groups. This research indicates that chemical modification strategies can lead to significant improvements in the properties of lignin-based polymeric materials using a higher fraction of an inexpensive lignin monomer from renewable resources and a lower fraction an expensive, petroleum-derived isocyanate monomer to achieve the required material properties.

KEYWORDS: renewable resources, lignin, Lewis acid, polyurethane

\section{INTRODUCTION}

Lignin is a highly abundant natural polymer composed of three different types of phenylpropane units that form a rigid, threedimensional network. ${ }^{1-3}$ In spite of high availability of this feedstock material, there are few lignin-based commodity materials because of its complex structure, low reactivity, broad chemical differences depending on source, and poor processability. Lignin has been studied for two general uses; the first is production of small molecule chemicals by decomposition or refinery process. Catalytic methods used to convert lignin include various types of reduction, oxidation, and catalytic cracking process. ${ }^{4-7}$ Because lignin is an aromatic macromolecule derived from plants, this is an ideal strategy to substitute petroleum-based feedstock for those based on renewable resources. Typically, softwood lignin has been actively researched by many researchers to produce industrially important vanilline due to its structural similarity with that of lignin. ${ }^{8,9}$ Also, many other industrially important small molecule chemicals can be produced by catalytic decomposition of lignin. ${ }^{4,8,9}$ However, additional research is needed to overcome serious practical issues associated with the huge energy costs and necessary purification process to produce small molecule chemicals from this complex natural material. The second approach to the general use of lignin is as a starting material of a diversity of commercial polymer products. ${ }^{10-13}$ However, current commercial polymers require high-purity

Received: March 9, 2012

Accepted: May 10, 2012

Published: May 10, 2012 
starting materials having significant and reproducible reactivity. Beyond the variability intrinsic to lignin derived from different sources or processing methods, lignin also changes its properties depending on humidity, temperature and UV exposure. ${ }^{14}$ Therefore, some degree of controlled modification in lignin is necessary process to produce commercial products. $^{10,15,16}$

Although the general lignin framework is based on ether and aliphatic linkages, lignin contains various functional groups such as hydroxyl, methoxy, carboxylic acid, ether, aldehyde, ethylenic, and ester groups. ${ }^{1-3,10}$ Among various functional groups in lignin, hydroxyl group and methoxy group are found in high concentrations but this can be strongly affected by the subsequent processing steps. ${ }^{2,17}$ There were a few studies of ether cleavage and demethylation of methoxy groups using hydrogen iodide, ${ }^{18}$ hydrogen chloride ${ }^{19,20}$ and other diverse hydrolysis reactions in acidic conditions; ${ }^{21-23}$ however, many of these results were inconclusive due to nonquantitative characterization of material changes.

There have been a few studies on demethylating lignin using industrially relevant processes. An et al. ${ }^{24,25}$ and $\mathrm{Wu}$ et al. ${ }^{25,26}$ studied the use of sulfur dioxide-mediated demethylation in converting methoxy groups to hydroxyl groups. They demonstrated that a $40 \%$ reduction in methyl group content can be achieved, and the resultant material appeared to be suitable for use in formaldehyde-based adhesive resins. However, it is known that $\mathrm{SO}_{2}$ treatments can result in significant increases in sulfonate groups, ${ }^{27}$ which can reduce material stability in water-contacting applications. Alternatives to these approaches should increase lignin reactivity while avoiding these types of side reactions.

The demethylation of methoxy groups using Lewis acids has been extensively studied in small molecule organic chemistry. ${ }^{28}$ The methoxy group in lignin can be converted to a more reactive hydroxyl group via demethylation..$^{10,14-16}$ In this report, hydrobromic acid $(\mathrm{HBr})^{29-32}$ treatment was found to be effective in increasing lignin hydroxyl-group content and reactivity, while other Lewis acids like boron tribromide $\left(\mathrm{BBr}_{3}\right),{ }^{31,33-35}$ and trimethylsilyl iodide (TMSI) $)^{34,36,37}$ were not. Determination of chemical changes in lignin was followed with ${ }^{1} \mathrm{H}$ NMR spectroscopy. ${ }^{38-43}$ Because it is difficult to directly measure hydroxyl groups using ${ }^{1} \mathrm{H}$ NMR, hydroxyl groups was acetylated to acetoxy groups prior to a ${ }^{1} \mathrm{H}$ NMR characterization. ${ }^{39,42,44}$ In this report, the modified lignin was characterized by ${ }^{1} \mathrm{H}$ NMR and then the amount of hydroxyl groups was measured based upon the acetoxy group amount. Also, molecular weight distribution and qualitative measures of hydrophilicity of modified and unmodified lignin were determined using gel permeation chromatography (GPC) and water contact angle test, respectively.

Polyurethanes are a widely used polymer materials because they can be processed to versatile forms such as solid elastomers, fibers, foams, solid plastics, and coating and adhesive materials. ${ }^{45}$ Many commercial polyurethane formulations find application as biocompatible materials in medical devices. ${ }^{46}$ Most are prepared from the formation of urethane linkages by the reaction of isocyanates and polyols. ${ }^{47}$ Because lignin can contain high concentrations of hydroxyl groups on an aromatic macromolecule, lignin can function as polyols to form polyurethane network structure. Lignin has been shown to form thermoset materials when combined with synthetic polyols to provide a low-cost component based on renewable resources. ${ }^{10,11,48-53}$ Structure-property relationships of lignin- based polyurethanes indicated that higher densities of network from high cross-linking degree influenced the mechanical

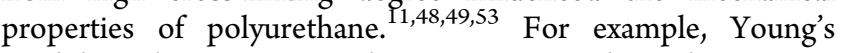
modulus, ultimate stress, ultimate strain, and toughness were sensitively affected by isocyanate and hydroxyl group stoichiometry, which can govern by the cross-linking density in the presence of lignin. The cross-linking density also affects thermal behavior, such as the glass transition temperature, and the swelling ratio of synthesized polyurethanes as well. In this report, correlations between changes in hydroxyl group content and material properties in cross-linked polyurethane materials made from native softwood Kraft lignin and Lewis acidmodified softwood lignin will also be presented.

\section{EXPERIMENTAL SECTION}

Lewis-Acid-Catalyzed Lignin Modification. Lignin was purchased from TCI America (TCI product number: L0082, softwood lignin) and used after repeated washing with aqueous $2 \mathrm{M}$ hydrogen chloride $(\mathrm{HCl})$ solution as described elsewhere. ${ }^{54}$ All other reagents were purchased from Aldrich Chemical Co. and used without further purification. Lignin $(500 \mathrm{mg})$ was dissolved in $2 \mathrm{~mL}$ of DMF prior to addition of $2.5 \mathrm{~g} \mathrm{48 \%}$ aqueous hydrobromic acid and hexadecyltributylphosphonium bromide (TBHDPB). The reaction mixture was stirred for $20 \mathrm{~h}$ at $115^{\circ} \mathrm{C}$. Then the ambient temperaturecooled reaction mixture was added dropwise into $250 \mathrm{~mL}$ of 2 $\mathrm{M}$ aqueous $\mathrm{HCl}$ solution. The precipitate was vigorously stirred for $3 \mathrm{~h}$ before filtration. The heterogeneous solution containing solid precipitate filtered through fritted disk funnels to isolate solid particles from the solution. The obtained solid powder was washed with water and then dried overnight in a vacuum oven at $40{ }^{\circ} \mathrm{C}$. The dried solid powder was suspended in diethyl ether and vigorously stirred for $10 \mathrm{~min}$ to purify it. The solid powder product was recovered and dried under high vacuum overnight at $40{ }^{\circ} \mathrm{C}$. Other demethylation reactions were attempted with $\mathrm{BBr}_{3}$ and TMSI instead of $\mathrm{HBr} / \mathrm{TBHDPB}$ at room temperature for 40 and $50 \mathrm{~h}$, respectively. These other procedures were the same as for $\mathrm{HBr} / \mathrm{TBHDPB}$.

Acetylation and ${ }^{1} \mathrm{H}$ NMR Characterization of Unmodified/Modified Lignin. Lignin $(200 \mathrm{mg})$ was dissolved in $4 \mathrm{~mL}$ pyridine to form a homogeneous solution. Then $4 \mathrm{~mL}$ of acetic anhydride was added and stirred for $48 \mathrm{~h}$ at room temperature. The crude reaction solution was added dropwise and precipitated in cold water and then filtered through fine pore size fritted disk funnel. The resulting solid product was washed with water until acetic acid removed. The final product was dried overnight in the vacuum oven at $40{ }^{\circ} \mathrm{C}$. ${ }^{1} \mathrm{H}$ NMR spectra were recorded on a Bruker Advance 300 spectrometer in DMSO- $\mathrm{d}_{6}$ at room temperature for 128 scans. Pentafluorobenzaldehyde (PFB) $(10 \mu \mathrm{L})$ was added as an internal standard for each sample.

Modified/unmodified lignin was dissolved in DMSO- $\mathrm{d}_{6}$ to obtain ${ }^{1} \mathrm{H}$ NMR spectra without acetylation. Methoxy group concentration was measured to determine hydroxylation rate.

Lignin Film Preparation and Water Contact Angle Measurement. Lignin (40 mg) was dissolved in DMF (0.2 $\mathrm{mL}$ ) until the reaction mixture became homogeneous. The lignin solution $(50 \mu \mathrm{L})$ was spin-coated on $1.5 \mathrm{~cm} \times 1.5 \mathrm{~cm}$ slide glass at $1000 \mathrm{rpm}$ for $60 \mathrm{~s}$. The lignin coated-glass was dried in vacuum oven for $24 \mathrm{~h}$ at $60{ }^{\circ} \mathrm{C}$ to remove solvent. The dried lignin film coated glasses were preconditioned for $3 \mathrm{~h}$ under the environmental conditions of measurement. Water droplets $(10 \mu \mathrm{L})$ were placed on the surface of the lignin film 
Scheme 1. Lewis-Acid-Catalyzed Lignin Demethylation and Proposed Synthesis of Lignin-Based Polyurethane from Demethylated $\operatorname{Lignin}^{a}$

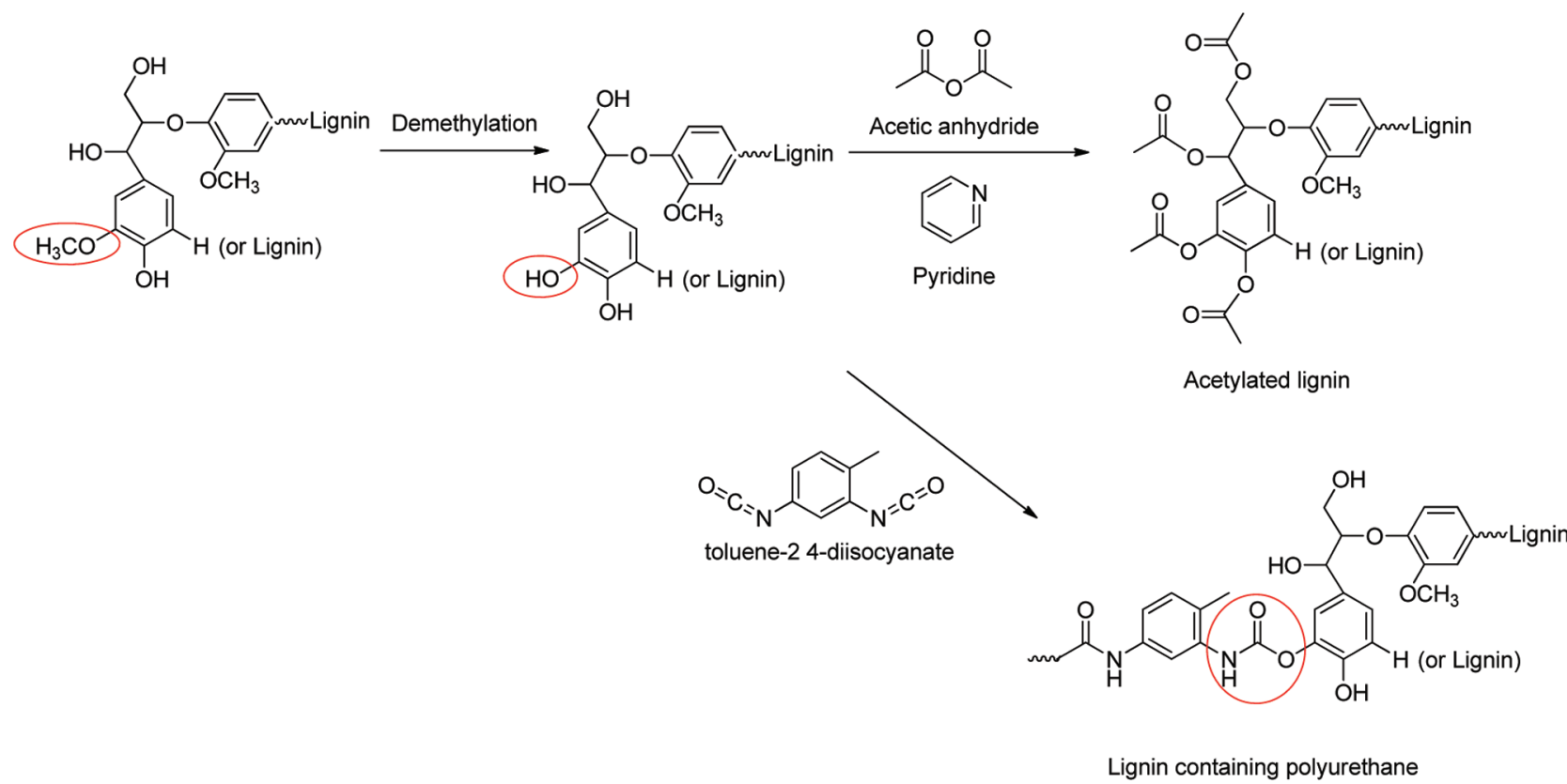

${ }^{a}$ Demethylated lignin was subsequently acetylated with acetic anhydride and pyridine to convert hydroxyl groups to acetoxy groups. Lignin-based polyurethane was prepared by condensation reaction between lignin and toluene-2,4-diisocyanate. The reaction scheme of lignin hydroxylations represents an example of a possible general demethylation process, not a specific, selective reaction.

and then imaged after $20 \mathrm{~s}$. Ten measurements were carried out and the average and standard deviation of the measurements calculated.

Preparation of Unmodified/Modified Lignin-Based Polyurethane. Polyurethanes were prepared through condensation reactions between diisocyanate in toluene-2,4diisocyanate (TDI) and hydroxyl groups in lignin. Then polyethylene glycol (PEG; $200 \mathrm{~g} / \mathrm{mol}$ ), was used to as the soft segment in polyurethane structure to avoid brittleness of final product. To form polyurethanes using $\mathrm{HBr}$-modified lignin, $200 \mathrm{mg}$ of lignin was mixed with TDI $(500 \mathrm{mg})$ at $75^{\circ} \mathrm{C}$ and prepolymerized for $5 \mathrm{~min}$. PEG $200(450 \mathrm{mg}$ ) was mixed to previously prepared lignin-TDI mixture and the reaction mixture was then mechanically stirred for $5 \mathrm{~min}$ at $75{ }^{\circ} \mathrm{C}$ followed by final polymerization at $75^{\circ} \mathrm{C}$ for additional $2 \mathrm{~h}$. All polyurethane preparation processes were performed in custom mold with a $20 \mathrm{~mm}$ diameter and $10 \mathrm{~mm}$ thickness to make a disk-shaped testing specimen. Unmodified lignin-based polyurethane and $\mathrm{HBr}$-modified lignin-based polyurethane were prepared under the exactly same polymerization conditions. Pure polyurethane was prepared without lignin addition under the same polymerization conditions as above-described.

Mechanical Property Testing (Compression Test). Uniaxial stress-stain behaviors of lignin-based polyurethane samples were measured with an Instron testing machine (model 4400R). Load was recorded as a function of displacement with a crosshead speed of $1 \mathrm{~mm} / \mathrm{min}$ and then the obtained data was converted to stress-strain plot. Mechanical properties, including initial modulus and stress at $10 \%$ strain, were calculated from the stress-strain plots. Tests were performed at least 4 times for each type of material and data points were averaged with standard deviation.

Scanning Electron Microscope (SEM) Analysis of Lignin and Lignin-Based Polyurethane. Dried specimens were attached to SEM stubs using double-stick tape, and then coated with gold using a Pelco SC-6 sputter coater. The specimens were examined using a Hitachi $2460 \mathrm{~N}$ scanning electron microscope (SEM). Digital images were obtained using Quartz PCI Image management system software for detail analysis of image.

\section{RESULTS AND DISCUSSION}

Lewis-Acid-Catalyzed Modification of Lignin. As shown in scheme 1 , ether cleavage and demethylation were expected to target aromatic and aliphatic methoxy groups in lignin. Three common demethylation strategies in small molecule synthesis were tested to perform demethylation in lignin..$^{10,14,16,28-37}$ In addition, some ether linkages in lignin may have been involved in the Lewis-acid-catalyzed ether cleavage reaction. The demethylation method was carried out in $48 \%$ aqueous $\mathrm{HBr}$ in the presence of phase-transfer agent, TBHDPB, in a waterDMF solvent mixture. ${ }^{29-32}$ The reaction mixture was heated at $115{ }^{\circ} \mathrm{C}$ for $20 \mathrm{~h}$ with vigorous stirring. After the designated reaction time, the reaction mixture was precipitated in water. The solid powder formed was isolated from the solution for drying and then purified once more by washing with diethyl ether. ${ }^{31,33-37}$

Figure 1c shows broad proton signal at $\delta$ 7.7-6.0 corresponding to protons in aromatic groups and $\delta 4.8-3.0$ corresponding to protons in aliphatic/aromatic methoxy group. Proton signals from hydroxyl groups were not always clearly visible in the spectra, possibly due to hydrogen bonding. 55 Therefore, we reacted lignin via pyridine-catalyzed acetylation with acetic anhydride to convert hydroxyl groups to acetoxy groups. ${ }^{38-44}$ Integrals of protons at acetoxy groups are observed between at chemical shifts of $1.58-2.70$ in panels a and $b$ in Figure 1. PFB was added as an internal standard, the peaks for which appear at $\delta 10.14$ for each spectrum. PFB was 


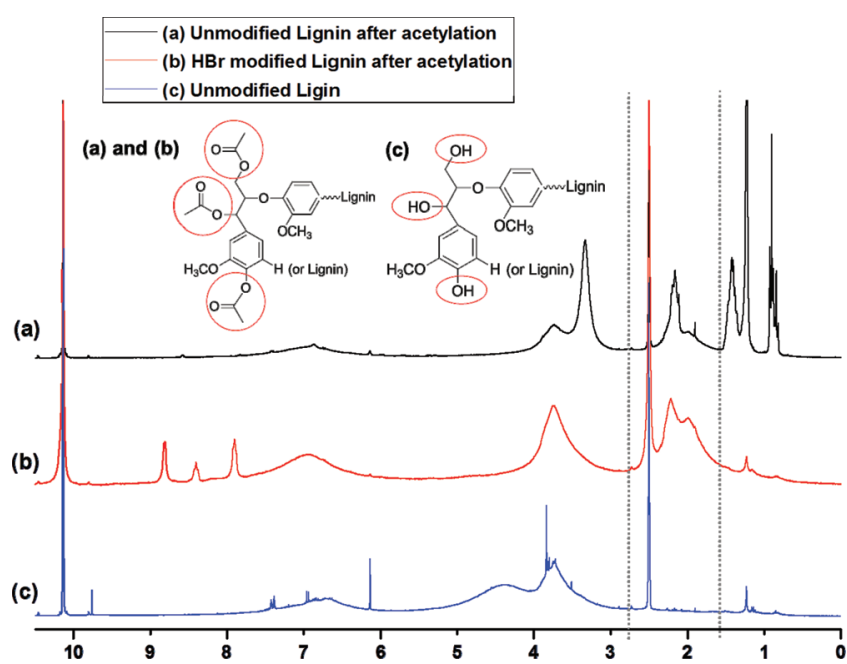

Figure 1. ${ }^{1} \mathrm{H}$ NMR spectra of (a) HBr-catalyzed modified lignin after acetylation, (b) unmodified lignin after acetylation, and (c) unmodified lignin. Acetoxy group area at $\delta 2.70-1.58$ correspond to the concentration of hydroxyl groups in modified lignin. (a) $\mathrm{HBr}$ modified lignin and (b) ummodified lignin were represented in a general structure, but each lignin has different concentrations of acetoxy groups in a unit mass. Dotted lines indicate integration ranges of acetoxy group.

chosen because of three reasons: first, it is very well miscible in the ${ }^{1} \mathrm{H}$ NMR sample solutions. Second, PFB is easy to handle because it is not volatile. Third, PFB has its proton signals far from the major signals of the major lignin products.

Hydroxyl group concentration in lignin was also measured by comparing methoxy group area at $\delta 4.8-3.0$ without acetylation or other further chemical modification of lignin. Because Lewis acid modification of lignin did not appear to affect aromatic groups, the aromatic group proton signal at $\delta 7.7-6.0$ was used as an internal standard. The conversion of methoxy group functionality to hydroxyl group after Lewis acid modification can be easily characterized by decreases in methoxy group area at $\delta$ 4.8-3.0. The methoxy group decrease after Lewis acid modification showed the same changing rate as acetoxy group measurement after acetylation modification. In this report, we used acetoxy group measurements after acetylation of lignin because this method is a more commonly used hydroxyl group characterization method in lignin modification. . $^{3,42,44}$

Each measured hydroxyl group concentration was normalized by sample weight for NMR characterization. Lewis acid catalyzed modifications were performed at least three times. $\mathrm{HBr}$ reaction resulted in measurable changes in hydroxyl content with a $28 \%$ increase; however, TMSI and $\mathrm{BBr}_{3}$ did not show significant change after the modification reaction (data not shown). According to GPC analysis of pre- and post- $\mathrm{HBr}$ catalyzed modified lignin, no significant changes in molecular weight and molecular weight distribution occurred in any of the reactions. Detailed experimental results and analysis are discussed in the Supporting Information.

Concentration of hydroxyl group is expected to affect hydrophilicity of lignin, and changes in the hydrophilicity of the lignin could be assessed qualitatively by water contact angle measurements. Unmodified lignin and $\mathrm{HBr}$-modified lignin were dissolved in DMF and then they were spin-coated on glass slides. Residual DMF was removed in a vacuum oven prior to water contact angle measurements. Figure 2 (a) is a side view of water contact angle image on unmodified lignin film and (b) is
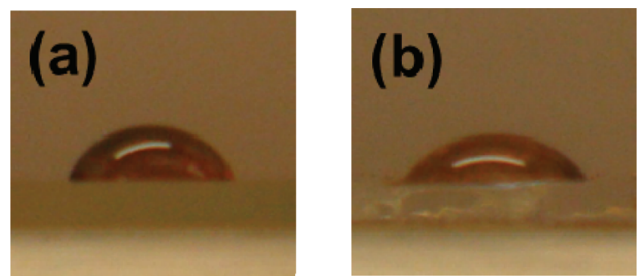

Figure 2. Water contact angles of (a) unmodified-lignin film $\left(63.8^{\circ} \pm\right.$ $\left.3.8^{\circ}\right)$ and $(\mathrm{b}) \mathrm{HBr}$ modified-lignin film $\left(46.5^{\circ} \pm 1.5^{\circ}\right)$ on glass surface. Water contact angle measurements were taken across the sample and are an average of 10 measurements.

$\mathrm{HBr}$ modified lignin. Water contact angle changed from $63.8^{\circ}$ $\pm 3.8^{\circ}$ to $46.5^{\circ} \pm 1.5^{\circ}$ after $\mathrm{HBr}$ modification of lignin. The water contact angle decrease indicated an increased hydrophilicity of the material surface, which is attributed to increases in hydroxyl group concentration in lignin. The water contact angle measurements were further indication that $\mathrm{HBr}$ modification of lignin is an effective technique that could be used to adjust lignin chemical functionality. In this respect, the modified lignin could be a useful new biodegradable and renewable plant-based coating material with a tuned surface hydrophilicity.

HBr-Modified Lignin-Based Polyurethane. The material properties of polyurethanes are governed by the ratio of hard and soft segments. Although the contribution of lignin to the mechanical properties is thought to be as a hard segment, in terms of reactivity, lignin will have the role of polyol in polyurethane synthesis. In lignin-polymer blends, solubility parameters of various polymers and lignin are an factor to determine miscibility and properties of lignin-polymer blend products. ${ }^{56}$ However, the covalently cross-linked lignin-based polyurethane was more affected by degree of cross-linking and a chemical structure of cross-linkers. There have been extensive investigations in lignin-based polyurethane to find a relationship between various ratios of isocyanate/polyols and resultant material properties. ${ }^{45,48-53,57}$ In the present study, two different polyurethanes were prepared to study their property difference between $\mathrm{HBr}$-modified lignin, which has high hydroxyl group contents in unit weight, and unmodified lignin.

Polyurethanes were prepared from reaction of TDI and lignin as a polyol. ${ }^{48,49}$ Polyethylene glycol, which had numberaverage molecular weight of $200 \mathrm{~g} / \mathrm{mol}$ (PEG 200), was used as a linear polyol comonomer to increase elasticity of the final polyurethane product. Polyurethanes formed from the reaction between TDI and lignin were too brittle without PEG 200 and fractured easily upon handling (data not shown). SEM images of cross sections of prepared polyurethanes with $40 \%$ PEG are shown in figure 3. Both polyurethanes had similar porous structures, with pore sizes ranging up to $1 \mathrm{~mm}$, suggesting that any changes in mechanical properties would be attributed to changes in the included materials not microstructure.

$\mathrm{HBr}$-modified lignin showed significant mechanical-property improvement over both polyurethanes containing unmodified lignin and pure polyurethane that does not include lignin. The mechanical properties of lignin-based polyurethanes and pure polyurethanes were measured by compression testing. Initial modulus and stress at $10 \%$ strain were calculated from stressstrain curves. Typical stress-strain behaviors of polyurethane for unmodified lignin-based polyurethane and $\mathrm{HBr}$-modified lignin-based polyurethane are shown in Figure 4. In comparing these two stress-strain behaviors, $\mathrm{HBr}$-modified lignin-based 

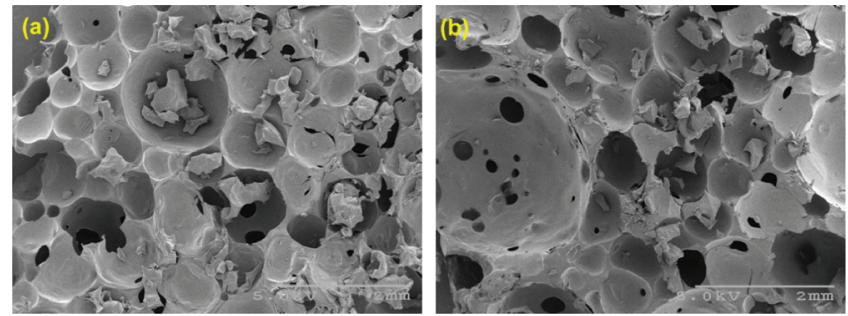

Figure 3. Scanning electron microscope (SEM) images of crosssections of lignin-based polyurethane. (a) $\mathrm{HBr}$-catalyzed hydroxylated lignin-based polyurethane and (b) unmodified native lignin-based polyurethane.

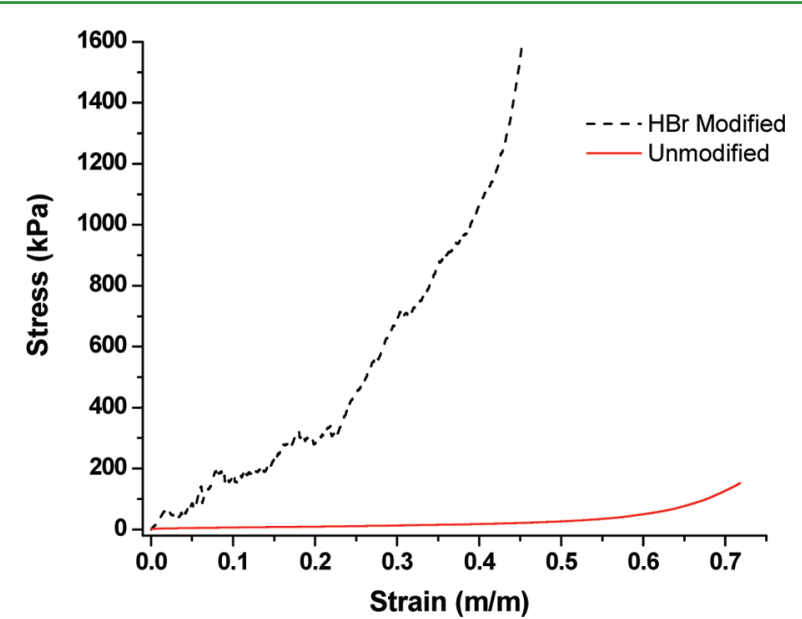

Figure 4. Compression test stress-strain relationships of $\mathrm{HBr}$ modified lignin-based polyurethane (black dashed line) and unmodified lignin-based polyurethane (red solid line); two different characters of mechanical properties, i.e., initial modulus and stress at $10 \%$ strain were obtained from the stress-strain curve.

polyurethane revealed superior stress values over the whole strain range; this result demonstrates that $\mathrm{HBr}$-modified ligninbased polyurethane is much stronger material than unmodified sample. Figure 5 summarizes mechanical property parameters of unmodified lignin-based polyurethane, modified lignin-based polyurethane and pure polyurethane. The presented data are average values with standard deviations based on at least three separate measurements. Initial moduli and stresses at $10 \%$ strain were normalized by density of each sample.

Lignin-based polyurethane were expected to form crosslinked network structures due to multiple hydroxyl group functionality on lignin. Therefore, if there were more hydroxyl groups in lignin then the final polyurethane will have denser network structure, which results higher mechanical property, than less hydroxylated lignin. Estimates of cross-link densities of lignin-based polyurethane were determined from materials' moduli information..$^{58-60}$ In rubber elasticity theory, the number of network strands per unit volume $(N$, effective cross-link density) can be obtained from the relation, $E=$ $3 N k_{\mathrm{B}} T$, where $k_{\mathrm{B}}$ is Boltzmann's constant $\left(1.38 \times 10^{-23} \mathrm{~J} / \mathrm{K}\right)$ and $T$ is the absolute temperature in Kelvin. To calculate the effective cross-linking density, $N$, we obtained each sample's modulus $(E)$ from mechanical property test as shown in Figures 4 and 5 . The determined average $N$ were $9.97 \times 10^{21} \mathrm{~m}^{-3}$ and $9.52 \times 10^{22} \mathrm{~m}^{-3}$ for each unmodified lignin-based polyurethane and $\mathrm{HBr}$-modified lignin-based polyurethane, respectively. As

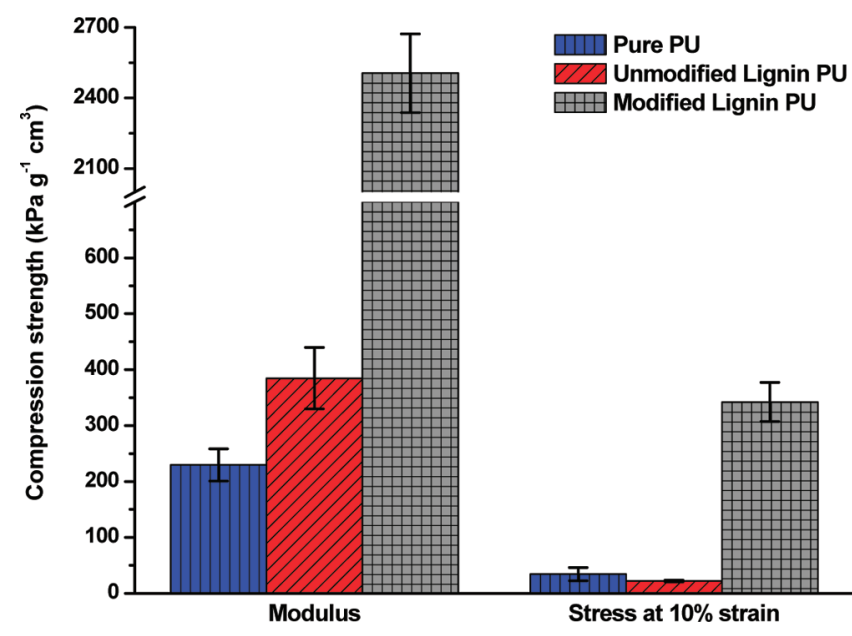

Figure 5. Summary of mechanical properties of lignin-based polyurethane obtained from stress-strain relationship of material. Pure polyurethane without lignin (blue vertical line pattern), unmodified lignin-based polyurethane (red oblique line pattern), and HBr-modified lignin-based polyurethane (gray square pattern) were compared to demonstrate initial modulus and stress at $10 \%$ strain. Compression strength $(\mathrm{kPa})$ was normalized by density of samples $(\mathrm{g} /$ $\left.\mathrm{cm}^{3}\right)$.

we hypothesized, HBr-modified lignin-based polyurethane had a significantly higher cross-link density compare to unmodified.

In this lignin-based polyurethane mechanical property tests as shown in Figure 5, HBr-modified lignin-based polyurethane showed overall improved mechanical properties compare to unmodified lignin-based polyurethane and pure polyurethane. Pure polyurethane had a density-normalized initial modulus of $229.62 \pm 28.88 \mathrm{kPa} \mathrm{g}^{-1} \mathrm{~cm}^{3}$ and a density-normalized stress at $10 \%$ strain of $34.44 \pm 11.85 \mathrm{kPa} \mathrm{g}^{-1} \mathrm{~cm}^{3}$. Unmodified ligninbased polyurethane had density-normalized initial modulus of $384.77 \pm 54 \mathrm{kPa} \mathrm{g}^{-1} \mathrm{~cm}^{3}$ and $21.98 \pm 1.6 \mathrm{kPa} \mathrm{g}^{-1} \mathrm{~cm}^{3}$ of density-normalized stress at $10 \%$ strain. $\mathrm{HBr}$-modified ligninbased polyurethane had the highest values for these metrics with $2505.40 \pm 167 \mathrm{kPa} \mathrm{g}^{-1} \mathrm{~cm}^{3}$ of density-normalized initial modulus and $342.14 \pm 34.6 \mathrm{kPa} \mathrm{g}^{-1} \mathrm{~cm}^{3}$ of density-normalized normalized stress at $10 \%$ strain. The improved mechanical properties of $\mathrm{HBr}$-modified lignin-based polyurethane suggests that high cross-linking degree polyurethane structure from high concentration of hydroxyl groups significantly contribute to mechanical property improvements in polyurethanes. ${ }^{61,62}$ In the same principle, pure polyurethane that does not include any lignin showed the lowest modulus because of the lowest degree of cross-linking. Likewise, both pure polyurethane and ligninbased polyurethane displayed substantially lower stresses at $10 \%$ strain than $\mathrm{HBr}$-modified lignin-based polyurethane. The both values are very low and close each other. This result suggest that in the case of poorly cross-linked polyurethane, slight difference of cross-linking degree does not give a substantial differences in certain mechanical property, stress at $10 \%$ strain. The included lignin in the polyurethane has a role of fillers in addition to a role of polyol cross-linking agent. If unmodified lignin forms a weakly linked filler phase in the polyurethane, increasing hydroxyl group content could be expected to form physically cross-linked secondary interactions between polymer chains and improve mechanical properties. ${ }^{61,62}$ Overall, the mechanical property improvement of polyurethane demonstrates an example of how chemical 
modification strategies of natural materials can effect improvement of commodity polymer properties.

\section{CONCLUSION}

This report presents: first, successful functional group modification of renewably sourced material, lignin, using selected Lewis acid catalyst without detrimental side reactions, and second, two representative lignin-containing materials, lignin-coated surfaces and lignin-based polyurethanes, to demonstrate the importance of lignin chemical modification in improving material properties. In the presented Lewis acid catalyst system, $\mathrm{HBr}$ /TBHDPB increased the hydroxyl groups of lignin by $28 \%$ in average. To perform a quantified characterization of hydroxyl groups in lignin, we acetylated the hydroxyl groups prior to ${ }^{1} \mathrm{H}$ NMR analysis. High concentration of hydroxyl groups in $\mathrm{HBr}$-modified lignin increased surface hydrophilicity by $27 \%$ in water-contact-angle measurement of lignin-coated materials. Synthesized $\mathrm{HBr}-$ modified lignin-based polyurethane showed highly enhanced mechanical properties because of high cross-linking density. $\mathrm{HBr}$-modified lignin-based polyurethane has 6.5-folds higher initial modulus and 15.5-folds higher stress at $10 \%$ strain than unmodified lignin-based polyurethane.

\section{ASSOCIATED CONTENT}

\section{S Supporting Information}

Additional figures and information (PDF). This information is available free of charge via the Internet at http://pubs.acs.org/.

\section{AUTHOR INFORMATION}

\section{Corresponding Author}

*E-mail: washburn@andrew.cmu.edu.

\section{Present Address}

${ }^{\ddagger}$ Division of Chemistry and Chemical Engineering, California Institute of Technology, Pasadena, CA.

Notes

The authors declare no competing financial interest.

\section{ACKNOWLEDGMENTS}

The authors would like to thank Joseph Prata and Allison Elder for help with mechanical testing.

\section{REFERENCES}

(1) Heitner, C.; Dimmel, D. R.; Schmidt, J. A. Lignin and Lignins: Advances in Chemistry; CRC Press: Boca Raton, FL, 2010.

(2) Chakar, F. S.; Ragauskas, A. J. Ind. Crop Prod. 2004, 20, 131-141.

(3) Adehsives from Renewable Resources; Hemignway, R. W., Conner, A. H., Branham, S. J., Eds.; American Chemical Society: Washington, D.C., 1989; Vol. 385.

(4) Zakzeski, J.; Bruijnincx, P. C. A.; Jongerius, A. L.; Weckhuysen, B. M. Chem. Rev. 2010, 110, 3552-3599.

(5) Herrmann, W. A.; Weskamp, T.; Zoller, J. P.; Fischer, R. W. J. Mol. Catal. A: Chem. 2000, 153, 49-52.

(6) Pardini, V. L.; Vargas, R. R.; Viertler, H.; Utley, J. H. P. Tetrahedron 1992, 48, 7221-7228.

(7) Crestini, C.; Pastorini, A.; Tagliatesta, P. J. Mol. Catal. A: Chem. 2004, 208, 195-202.

(8) Rodriques, A. E.; da Silva, E. A. B.; Zabkova, M.; Araujo, J. D.; Cateto, C. A.; Barreiro, M. F.; Belgacem, M. N. Chem. Eng. Res. Des. 2009, 87, 1276-1292.

(9) Doherty, W. O. S.; Mousavioun, P.; Fellows, C. M. Ind. Crop Prod. 2011, 33, 259-276.
(10) Meister, J. J. In Polymer Modification; Principles, Techniques, and Applications; Meister, J. J., Ed.; Marcel Dekker: New York, 2000, p 67144.

(11) Wang, J. S.; Manley, R. S.; Feldman, D. Prog. Polym. Sci. 1992, 17, 611-646.

(12) Satyanarayana, K. G.; Arizaga, G. G. C.; Wypych, F. Prog. Polym. Sci. 2009, 34, 982-1021.

(13) Meister, J. J.; Patil, D. R.; Field, L. R.; Nicholson, J. C. J. Polym. Sci. Polym. Chem. 1984, 22, 1963-1980.

(14) Allan, G. G. In Lignins; Sarkanen, K. V., Ludwig, C. H., Eds.; John Wiley \& Sons: New York, 1971; pp 511-573.

(15) Meister, J. J. In Polymer Modification; Principles, Techniques, and Applications; Meister, J. J., Ed.; Marcel Dekker: New York, 2000, p 129-157.

(16) Meister, J. J. J. Macromol. Sci., Polym. Rev. 2002, C42, 235-289.

(17) Alen, R. In Papermaking Science and Technology; Stenius, P., Ed.; Finnish Paper Engineers'Association: , 2000; Vol. 3, p 12-54.

(18) Akim, L. G.; Shevchenko, S. M.; Zarubin, M. Y. Wood Sci. Technol. 1993, 27, 241-248.

(19) Freudenberg, K.; Belz, W.; Niemann, C. Ber. Dtsch. Chem. Ges. 1929, 62, 1554-1561.

(20) Shorygina, N. N.; Grushnikov, O. P.; Tychina, V. D. Chemical Abstracts 1967, 67, 3862.

(21) Lai, Y. Z.; Guo, X. P. Holzforschung 1992, 46, 311-314.

(22) Leary, G. J.; Sawtell, D. A. Holzforschung 1984, 38, 53-54.

(23) Lundquist, K. Appl. Polym. Symp. 1976, 28, 1393-1407.

(24) An, X. N.; Schroeder, H. A.; Thompson, G. E. Chem. Ind. Prod. $1995,15,36-42$.

(25) Hu, L.; Pan, H.; Zhou, Y.; Zhang, M. Bioresources 2011, 6, 3515-3525.

(26) Wu, S. B.; Zhan, H. Y. Cellul. Chem. Technol. 2001, 35, 253262.

(27) Mohanty, A. K.; Kumar, M. N. S.; Erickson, L.; Misra, M. J. Biobased Mater. Biol. 2009, 3, 1-24.

(28) Bhatt, M. V.; Kulkarni, S. U. Synthesis 1983, 249-282.

(29) Hwang, K. J.; Park, S. Synth. Commun. 1993, 23, 2845-2849.

(30) Kojima, K.; Sawamoto, M.; Higashimura, T. Macromolecules 1991, 24, 2658-2662.

(31) Daly, W. H.; Moulay, S. J. Polym. Sci. Polym. Symp. 1986, 227242.

(32) Landini, D.; Montanari, F.; Rolla, F. Synthesis 1978, 771-773.

(33) Allan, G. G. Tappi 1968, 51, 224-225.

(34) Vickery, E. H.; Pahler, L. F.; Eisenbraun, E. J. J. Org. Chem. 1979, 44, 4444-4446.

(35) Westwood, G.; Horton, T. N.; Wilker, J. J. Macromolecules 2007, 40, 3960-3964

(36) Xiang, M. L.; Jiang, M.; Feng, L. X. Macromol. Rapid Commun. 1995, 16, 477-481.

(37) Yang, Z.; Pelton, R. Macromol. Rapid Commun. 1998, 19, 241246.

(38) Lundquist, K. In Methods in Ligin Chemistry; Lin, S. Y., Dence, C. W., Eds.; Springer-Verlag: Berlin, 1992; pp 242-249.

(39) Froass, P. M.; Ragauskas, A. J.; Jiang, J. E. Holzforschung 1998, $52,385-390$.

(40) Faix, O.; Argyropoulos, D. S.; Robert, D.; Neirinck, V. Holzforschung 1994, 48, 387-394.

(41) Tiainen, E.; Drakenberg, T.; Tamminen, T.; Kataja, K.; Hase, A. Holzforschung 1999, 53, 529-533.

(42) Lundquist, K. Acta Chem. Scand., B 1979, 33, 27-30.

(43) Lundquist, K.; Olsson, T. Acta Chem. Scand., B 1977, 31, 788792.

(44) Bonner, T. G.; Mcnamara, P. J. Chem. Soc., B 1968, 795-797.

(45) Alger, M. In Polymer Science Dictionary, 2nd ed.; Chapman \& Hall: London, 1997; pp 459-460.

(46) Saltzman, W. M. In Tissue Engineering; Oxford University Press: New York, 2004; pp 458-462.

(47) Odian, G. Principles of Polymerization, 4th ed.; John Wiley \& Sons: Hoboken, NJ, 2004. 
(48) Saraf, V. P.; Glasser, W. G. J. Appl. Polym. Sci. 1984, 29, 18311841.

(49) Saraf, V. P.; Glasser, W. G.; Wilkes, G. L.; Mcgrath, J. E. J. Appl. Polym. Sci. 1985, 30, 2207-2224.

(50) Saraf, V. P.; Glasser, W. G.; Wilkes, G. L. J. Appl. Polym. Sci. 1985, 30, 3809-3823.

(51) Hatakeyama, H.; Nakayachi, A.; Hatakeyama, T. Compoites, Part A 2005, 36, 698-704.

(52) Bonini, C.; D’Auria, M.; Ernanuele, L.; Ferri, R.; Pucciariello, R.; Sabia, A. R. J. Appl. Polym. Sci. 2005, 98, 1451-1456.

(53) Hatakeyama, T.; Asano, Y.; Hatakeyama, H. Macromol. Symp. 2003, 197, 171-180.

(54) Kim, Y. S.; Kadla, J. F. Biomacromolecules 2010, 11, 981-988.

(55) Silverstein, R. M.; Wester, F. X.; Kiemle, D. J. In Spectrometric Identification of Organic Compounds, 7th ed.; John Wiley \& Sons: New York, 2005; pp 150-155.

(56) Pouteau, C.; Baumberger, S.; Cathala, B.; Dole, P. C. R. Biol. 2004, 327, 935-943.

(57) Cateto, C. A.; Barreiro, M. F.; Rodrigues, A. E.; Brochier-Solan, M. C.; Thielemans, W.; Belgacem, M. N. J. Appl. Polym. Sci. 2008, 109, 3008-3017.

(58) Rubinstein, M.; Colby, R. H. In Polymer Physics; Oxford University Press: New York, 2003; pp 253-305.

(59) Chun, H.; Gent, A. N. J. Polym. Sci. Polym. Phys. 1996, 34, 2223-2229.

(60) Sekkar, V.; Narayanaswamy, K.; Scariah, K. J.; Nair, P. R.; Sastri, K. S.; Ang, H. G. J. Appl. Polym. Sci. 2007, 103, 3129-3133.

(61) Nielsen, L. E. J. Macromol. Sci. Rev. Macromol. Chem. 1969, C3, 69-103.

(62) Nielsen, L. E.; Landel, R. F. In Mechanical Properties of Polymers and Composites; Nielsen, L. E., Landel, R. F., Eds.; Marcel Dekker: New York, 1994; pp 233-336. 